\title{
ASYMPTOTIC BEHAVIOUR OF THE THREE-DIMENSIONAL $\alpha$-NAVIER-STOKES MODEL WITH LOCALLY LIPSCHITZ DELAY FORCING TERMS
}

\author{
T. CARABALLO, A.M. MÁRQUEZ-DURÁN, AND J. REAL
}

\begin{abstract}
We obtain some results on the existence and uniqueness, and exponential stability of solutions for the three-dimensional $\alpha$-Navier-Stokes model with delays, when the forcing term containing the delay is sub-linear and locally Lipschitz continuous.
\end{abstract}

\section{INTRODUCTION}

There exist several reasons which justify the study and importance of delay differential equations. For instance, if we want to model, in a realistic way, some evolution phenomena arising in Physics, Biology, Engineering, etc., some hereditary characteristics such as aftereffect, time lag, memory, and time delay should be considered in the variables appearing in the equations. We can mention some typical examples arising in the investigations of materials with thermal memory, in biochemical reactions, population models, etc. (see, for instance, Hale and Lunel [12], Murray [17], Ruess [18]-[19], Wu [21], Caraballo et al. [1], and the references therein).

On the other hand, delayed terms may appear when one tries to control a system by applying external forcing terms which take into account the history of the solution (see [6], [7], [20], for the case of Navier-Stokes equations with delays).

In our previous paper [5] we investigated the existence of stationary solutions of the so-called 3D $\alpha$-Navier-Stokes model when some terms, especially the external forcing one, contained some kind of globally Lipschitz nonlinear memory (e.g. delay) term. The non-delay version has received very much attention over the last years (see e.g. [10], [16], [11], [13] amongst others), and was originally intended as a closure model for the 3D turbulence averaged Reynolds model. The main reason is that this model has become very useful in order to approximate the 3D Navier-Stokes equations (notice that when $\alpha$ goes to zero, this problem converges to the usual 3D Navier-Stokes model). Also we investigated in [5] the exponential convergence of solutions to such stationary ones.

It is worth mentioning that, in order to circumvent serious difficulties in analyzing the three dimensional Navier-Stokes equations, there have been many modifications of them starting with Leray and mostly involving the nonlinear term, see the review

Key words and phrases. $\alpha$-Navier Stokes models with delays, existence and uniqueness of variational solutions, exponential convergence of solutions, asymptotic stability of stationary solutions.

Partially supported by Ministerio de Educación y Ciencia (Spain), FEDER (European Community) under grant MTM2005-01412, and Junta de Andalucía (Spain) under Proyecto de Excelencia P07-FQM-02468 and FQM314. 
paper of Constantin [9]. A system, called the globally modified Navier-Stokes equations (GMNSE), was introduced recently by Caraballo, Kloeden and Real [2, 3] and a similar analysis to the one carried out in the present paper is being investigated and will be reported elsewhere.

In this paper, we will be mainly concerned with the case in which the delay terms are only locally Lipschitz (as is considered in [20] for the 2D Navier-Stokes model with variable delay).

To start off, we first describe the details of our model. Let $\mathcal{O}$ be an open, bounded and connected subset of $\mathbb{R}^{3}$ with regular enough boundary $\partial \mathcal{O}$ (say $C^{2}$ for example), and consider some positive constants $\alpha, \nu$ and $h$. Denote by $A$ the Stokes operator and consider the problem

$$
\left\{\begin{array}{l}
\partial_{t}(u-\alpha \Delta u)+\nu(A u-\alpha \Delta(A u))+(u \cdot \nabla)(u-\alpha \Delta u) \\
-\alpha \nabla u^{*} \cdot \Delta u+\nabla p=F(t, u(t-\tau(t))), \text { in } \mathcal{O} \times(0,+\infty) \\
\nabla \cdot u=0, \text { in } \mathcal{O} \times(0,+\infty) \\
u=0, \quad A u=0, \quad \text { on } \partial \mathcal{O} \times(0,+\infty) \\
u(0)=u^{0}, \text { in } \mathcal{O}, \\
u=\phi, \text { in } \mathcal{O} \times(-h, 0)
\end{array}\right.
$$

where $u=\left(u_{1}, u_{2}, u_{3}\right)$ and $p$ are unknown fields defined in $\mathcal{O} \times[0,+\infty)$, representing, respectively, the large-scale (or averaged) velocity and the pressure, in each point of $\mathcal{O} \times[0,+\infty)$, of an incompressible viscous fluid with constant density filling the domain $\mathcal{O}$. The constants $\nu>0$ and $\alpha>0$ represent respectively the kinematic viscosity of the fluid, and the square of the spatial scale at which fluid motion is filtered. The term $F(t, u(t-\tau(t)))$ is an external force which eventually depends on the history of $u$, where $\tau(t) \geq 0$ denotes a variable delay. Finally, $u^{0}$ is a given initial velocity field, and $\phi$ is a given velocity field defined in $(-h, 0)$, where $h>0$ is a fixed time such that $\tau(t) \leq h$ for all $t \geq 0$. Notice that $u^{*}$ denotes the transpose of the vector $u$.

Although it is possible to analyse our model for a more general forcing term in (1) (for instance, for an abstract functional form as $F\left(t, u_{t}\right)$ where the notation $u_{t}$ refers to the segment of the solution $u$ defined for $s \in(-h, 0)$ as $\left.u_{t}(s)=u(t+s)\right)$ which could included a variety of delay terms in a unified formulation (as it was done in [5] in the globally Lipschitz case), we have chosen in this paper the particular case of variable delay previously mentioned in order to show, in a more clear way, the difficulties and differences with the globally Lipschitz situation considered in [5]. Moreover, one can also compare more easily with the assumptions and results in Taniguchi [20] for the case of 2D-Navier-Stokes.

The content of the paper is as follows. In Section 2 we formulate an abstract delay parabolic evolution equation which contains (1) as a particular case, and establish a result on existence and uniqueness of solutions. The stability properties of the zero solution for the abstract problem is then analysed in Section 3. Next, in Section 4, we first establish some sufficient conditions ensuring the existence (and eventual uniqueness) of stationary solutions for our abstract problem, and we then investigate the exponential convergence of solutions to the stationary ones. Finally, in Section 5, we prove that the previous abstract theory can be applied to our model (1). 


\section{Formulation as an abStract problem. Existence and uniqueness of SOLUTION}

Instead of working directly with the model (1), we will rewrite it in an abstract form, to establish a theory for this general model, and then to apply it to our particular situation. In this way, we can obtain more profit since the general theory can be applied to a variety of problems. First, we establish some notations and recall some properties of the nonlinear term $(u \cdot \nabla)(u-\alpha \Delta u)-\alpha \nabla u^{*} \cdot \Delta u$ appearing in the problem (1) (for a more detailed description see [8]).

2.1. Notation and properties of the nonlinear term. Denote by $(\cdot, \cdot)$ and $|\cdot|$, respectively, the scalar product and associate norm in $\left(L^{2}(\mathcal{O})\right)^{3}$, and by $(\nabla u, \nabla v)$ the scalar product in $\left(L^{2}(\mathcal{O})\right)^{3 \times 3}$ for the gradients of $u$ and $v$. Consider also the scalar product in $\left(H_{0}^{1}(\mathcal{O})\right)^{3}$ defined by

$$
((u, v))=(u, v)+\alpha(\nabla u, \nabla v), \quad \forall u, v \in\left(H_{0}^{1}(\mathcal{O})\right)^{3},
$$

where its associate norm, which is in fact an equivalent norm to the usual gradient one, will be denoted by $\|\cdot\|$.

Let $H$ be the closure in $\left(L^{2}(\mathcal{O})\right)^{3}$ of the following set

$$
\mathcal{V}=\left\{v \in(\mathcal{D}(\mathcal{O}))^{3}: \nabla \cdot v=0 \text { in } \mathcal{O}\right\},
$$

and let $V$ be the closure of $\mathcal{V}$ in $\left(H_{0}^{1}(\mathcal{O})\right)^{3}$. Then, $H$ is a Hilbert space for the inner product of $\left(L^{2}(\mathcal{O})\right)^{3}$, and $V$ is a Hilbert subspace of $\left(H_{0}^{1}(\mathcal{O})\right)^{3}$.

Denote by $A$ the Stokes operator defined by

$$
A w=-\mathcal{P}(\Delta w), \quad \forall w \in D(A),
$$

where $\mathcal{P}$ is the Leray operator, i.e., is the projector operator from $\left(\left(L^{2}(\mathcal{O})\right)^{3}\right.$ onto $H$. Taking into account that $\partial \mathcal{O}$ is regular enough, then $D(A)=\left(H^{2}(\mathcal{O})\right)^{3} \cap V$ and $|A w|$ defines a norm in $D(A)$ which is equivalent to the one in $\left(H^{2}(\mathcal{O})\right)^{3}$, in other words, there exists a constant $c_{1}(\mathcal{O})>0$ depending only on $\mathcal{O}$ such that

$$
\|w\|_{\left(H^{2}(\mathcal{O})\right)^{3}} \leq c_{1}(\mathcal{O})|A w|, \quad \forall w \in D(A) .
$$

For $u \in D(A)$ and $v \in\left(L^{2}(\mathcal{O})\right)^{3}$, we define $(u \cdot \nabla) v$ as the element in $\left(H^{-1}(\mathcal{O})\right)^{3}$ given by

$$
\langle(u \cdot \nabla) v, w\rangle_{-1}=\sum_{i, j=1}^{3}\left\langle\partial_{i} v_{j}, u_{i} w_{j}\right\rangle_{-1}, \quad \forall w \in\left(H_{0}^{1}(\mathcal{O})\right)^{3},
$$

where by $\langle\cdot, \cdot\rangle_{-1}$ we denote either the duality product between $\left(H^{-1}(\mathcal{O})\right)^{3}$ and $\left(H_{0}^{1}(\mathcal{O})\right)^{3}$, or between $H^{-1}(\mathcal{O})$ and $H_{0}^{1}(\mathcal{O})$.

On the other hand, if $u \in D(A)$, and $v \in\left(L^{2}(\mathcal{O})\right)^{3}$, we have that $\nabla u^{*} \cdot v \in$ $\left(L^{3 / 2}(\mathcal{O})\right)^{3} \subset\left(H^{-1}(\mathcal{O})\right)^{3}$, with

$$
\left\langle\nabla u^{*} \cdot v, w\right\rangle_{-1}=\sum_{i, j=1}^{3} \int_{\mathcal{O}}\left(\partial_{j} u_{i}\right) v_{i} w_{j} d x, \quad \forall w \in\left(H_{0}^{1}(\mathcal{O})\right)^{3} .
$$

It is not difficult to check that for $(u, w) \in D(A) \times D(A)$ and $v \in\left(L^{2}(\mathcal{O})\right)^{3}$, it follows

$$
\langle(u \cdot \nabla) v, w\rangle_{-1}=-\left\langle\nabla w^{*} \cdot v, u\right\rangle_{-1} .
$$


Consider now the trilinear form defined as

$$
b^{\#}(u, v, w)=\langle(u \cdot \nabla) v, w\rangle_{-1}+\left\langle\nabla u^{*} \cdot v, w\right\rangle_{-1},
$$

for all $(u, v, w) \in D(A) \times\left(L^{2}(\mathcal{O})\right)^{3} \times\left(H_{0}^{1}(\mathcal{O})\right)^{3}$. We then have the following result.

Proposition 1. ([8]) The trilinear form $b^{\#}$ satisfies

$$
b^{\#}(u, v, w)=-b^{\#}(w, v, u),
$$

for all $(u, v, w) \in D(A) \times\left(L^{2}(\mathcal{O})\right)^{3} \times D(A)$ and, consequently,

$$
b^{\#}(u, v, u)=0, \quad \forall(u, v) \in D(A) \times\left(L^{2}(\mathcal{O})\right)^{3} .
$$

Furthermore, there exists a constant $c(\mathcal{O})>0$, depending only on $\mathcal{O}$, such that

$$
\left|b^{\#}(u, v, w)\right| \leq c(\mathcal{O})|A u\|v \mid\| w \|,
$$

for all $(u, v, w) \in D(A) \times\left(L^{2}(\mathcal{O})\right)^{3} \times\left(H_{0}^{1}(\mathcal{O})\right)^{3}$, and

$$
\left|b^{\#}(u, v, w)\right| \leq c(\mathcal{O})\|u\||v||A w|,
$$

for all $(u, v, w) \in D(A) \times\left(L^{2}(\mathcal{O})\right)^{3} \times D(A)$.

2.2. Variational solution of (1). We now establish the assumptions on $F$ and the initial values $u^{0}$ and $\phi$, and we define the concept of variational solution.

Assume $F:[0,+\infty) \times V \rightarrow V$, satisfying:

d1) For all fixed $v \in V, F(\cdot, v)$ is measurable,

d2) There exists a function $g:[0,+\infty) \rightarrow[0,+\infty)$, with $g \in L^{p}(0, T)$ for all $T>0$, for some $1 \leq p \leq+\infty$, such that $\forall R>0, \exists L(R)>0$ : if $\|u\|,\|v\| \leq R$, then,

$$
\|F(t, u)-F(t, v)\| \leq L(R) g^{1 / 2}(t)\|u-v\|, \quad \forall t \geq 0,
$$

d3) For any $v \in V$,

$$
\|F(t, v)\|^{2} \leq g(t)\left(\|v\|^{2}+1\right), \quad \forall t \geq 0 .
$$

d4) Assume $u^{0} \in V, \phi \in L^{2 p^{\prime}}(-h, 0 ; V)$, where $\frac{1}{p}+\frac{1}{p^{\prime}}=1$.

As for the variable delay we suppose given a delay function $\tau \in C^{1}([0,+\infty))$ such that $0 \leq \tau(t) \leq h$ for all $t \geq 0$, and there exists a constant $\tau_{*}$ such that

$$
\frac{d \tau(t)}{d t}=\tau^{\prime}(t) \leq \tau_{*}<1, \quad \forall t \geq 0 .
$$

Definition 2. A variational solution for (1) is a function $u \in L^{2 p^{\prime}}(0, T ; D(A)) \cap$ $L^{2}(-h, T ; V) \cap L^{\infty}(0, T ; V)$ for all $T>0$, such that for all $w \in D(A)$,

$$
\begin{aligned}
&((u(t), w))+\nu \int_{0}^{t}(u(s)+\alpha A u(s), A w) d s+\int_{0}^{t} b^{\#}(u(s), u(s)-\alpha \Delta u(s), w) d s \\
&(11)=\left(\left(u^{0}, w\right)\right)+\int_{0}^{t}((F(s, u(s-\tau(s))), w)) d s, \quad t \geq 0,
\end{aligned}
$$

and coincides with $\phi$ in $(-h, 0)$.

Observe that (11) can be easily deduced from (1) by multiplying the first equation in (1) by $w \in D(A)$, taking into account the definition of the scalar product $((\cdot, \cdot))$, the expression of $b^{\#}$, and the equality (5).

Let us now rewrite (1) as an abstract problem. 
Set $\mathcal{H}:=V$, with the scalar product $(u, v)_{\mathcal{H}}=((u, v))$, and associated norm $|u|_{\mathcal{H}}=$ $\|u\|$, and $\mathcal{U}:=D(A)$, with the scalar product $((u, v))_{\mathcal{U}}=(A u, A v)$ and associated norm $\|u\|_{\mathcal{U}}=|A u|$. Then $\mathcal{H}$ and $\mathcal{U}$ are two real and separable Hilbert spaces such that $\mathcal{U} \subset \mathcal{H}$, being this injection compact and dense.

We identify $\mathcal{H}$ with its topological dual space $\mathcal{H}^{*}$, but considering $\mathcal{U}$ as a subspace of $\mathcal{H}^{*}$, where we identify $v \in \mathcal{U}$ with the element $f_{v} \in \mathcal{H}^{*}$ given by

$$
f_{v}(h)=(v, h)_{\mathcal{H}}, \quad \forall h \in \mathcal{H} .
$$

We denote by $\langle\cdot, \cdot\rangle$ the duality product between $\mathcal{U}^{*}$ and $\mathcal{U}$, and we define

$$
\langle\widetilde{A} u, v\rangle=\nu(A u, v)+\nu \alpha(A u, A v), \quad u, v \in D(A) .
$$

It is then clear that for any $v \in D(A)$,

$$
2\langle\widetilde{A} v, v\rangle=2 \nu(A v, v)+2 \nu \alpha(A v, A v) \geq 2 \nu \alpha|A v|^{2},
$$

and, if we denote by $\mu_{k}$ and $w_{k}, k \geq 1$, the eigenvalues and associate eigenvectors for the operator $A$, it can be checked that

$$
\left\langle\widetilde{A} w_{k}, v\right\rangle=\nu \mu_{k}\left(\left(w_{k}, v\right)\right) .
$$

Thus, taking

$$
\widetilde{\alpha}=2 \nu \alpha,
$$

we have:

a) $\widetilde{A} \in \mathcal{L}\left(\mathcal{U}, \mathcal{U}^{*}\right)$ is an operator such that

a1) $\widetilde{A}$ is self-adjoint,

a2) there exists $\widetilde{\alpha}>0$, such that

$$
2\langle\widetilde{A} v, v\rangle \geq \widetilde{\alpha}\|v\|_{\mathcal{U}}^{2}, \forall v \in \mathcal{U}
$$

Observe that the eigenvalues of the operator $\widetilde{A}$ are given by

$$
\lambda_{k}=\nu \mu_{k}
$$

On the other hand, let us define $\widetilde{B}: D(A) \times D(A) \rightarrow D(A)^{*}$, and $\widetilde{F}:[0,+\infty) \times V \rightarrow$ $V$ as

$$
\begin{gathered}
\langle\widetilde{B}(u, v), w\rangle=b^{\#}(u, v-\alpha \Delta v, w), \quad \forall(u, v, w) \in D(A) \times D(A) \times D(A), \\
\widetilde{F}(t, v)=F(t, v), \quad \forall(t, v) \in[0,+\infty) \times V
\end{gathered}
$$

Then, taking

$$
c_{1}=\frac{1+\alpha}{\alpha \nu} c_{1}(\mathcal{O}) c(\mathcal{O})
$$

we obtain that

b) $\widetilde{B}: \mathcal{U} \times \mathcal{U} \rightarrow \mathcal{U}^{*}$ is a bilinear mapping such that

b1) $\langle\widetilde{B}(u, v), u\rangle=0$, for all $u, v \in \mathcal{U}$,

b2) $\|\widetilde{B}(u, v)\|_{\mathcal{U}^{*}} \leq c_{1}|u|_{\mathcal{H}}\|v\|_{\mathcal{U}}$, for all $(u, v) \in \mathcal{U} \times \mathcal{U}$,

b3) $|\langle\widetilde{B}(u, v), w\rangle| \leq c_{1}\|u\|_{\mathcal{U}}\|v\|_{\mathcal{U}}|w|_{\mathcal{H}}$, for all $u, v, w \in \mathcal{U}$.

c) $\widetilde{F}:(0,+\infty) \times \mathcal{H} \longrightarrow \mathcal{H}$ is such that

c1) for any fixed $v \in \mathcal{H}, \widetilde{F}(\cdot, v)$ is measurable, 
c2) there exists a function $g:[0,+\infty) \rightarrow[0,+\infty)$, with $g \in L^{p}(0, T)$ for all $T>0$, for some $1 \leq p \leq+\infty$, such that $\forall R>0, \exists L(R)>0$ : if $|u|_{\mathcal{H}},|v|_{\mathcal{H}} \leq R$, then,

$$
|\widetilde{F}(t, u)-\widetilde{F}(t, v)|_{\mathcal{H}} \leq L(R) g^{1 / 2}(t)|u-v|_{\mathcal{H}} \quad \forall t \geq 0,
$$

c3) for any $u \in \mathcal{H}$,

$$
|\widetilde{F}(t, u)|_{\mathcal{H}}^{2} \leq g(t)\left(|u|_{\mathcal{H}}^{2}+1\right), \quad \forall t \geq 0 .
$$

d) $\phi \in L^{2 p^{\prime}}(-h, 0 ; \mathcal{H}), u^{0} \in \mathcal{H}$.

In this situation, given the initial data $u^{0} \in \mathcal{H}, \phi \in L_{\mathcal{H}}^{2 p^{\prime}}$, we can consider the problem

$$
\left\{\begin{array}{l}
u(t)+\int_{0}^{t} \widetilde{A} u(s) d s+\int_{0}^{t} \widetilde{B}(u(s), u(s)) d s \\
=u^{0}+\int_{0}^{t} \widetilde{F}(s, u(s-\tau(s))) d s, \forall t \geq 0, \\
u(t)=\phi(t), \quad \forall t \in(-h, 0) .
\end{array}\right.
$$

Definition 3. A solution of (17) is a function

$$
u \in L^{2 p^{\prime}}(-h, T ; \mathcal{H}) \cap L^{2}(0, T ; \mathcal{U}) \cap L^{\infty}(0, T ; \mathcal{H}) \text { for all } T>0,
$$

such that $u(t)$ coincides with $\phi(t)$ in $(-h, 0)$ and satisfies the equation from (17) in $\mathcal{U}^{*}$, for all $t \geq 0$.

Remark 4. Observe that if $u$ is a solution of (17) then $A u+B(u, u) \in L^{2}\left(0, T ; \mathcal{U}^{*}\right)$ for all $T>0$. Moreover, if we define $\tilde{g}(t)=g \circ \theta^{-1}(t)$, where $\theta:[0,+\infty) \longrightarrow$ $[-\tau(0),+\infty)$ is the differentiable strictly increasing function given by $\theta(s)=s-\tau(s)$, we obtain

$$
\begin{aligned}
\int_{0}^{T}|\widetilde{F}(t, u(t-\tau(t)))|_{\mathcal{H}}^{2} d t & \leq \int_{0}^{T} g(t)|u(t-\tau(t))|_{\mathcal{H}}^{2} d t+\int_{0}^{T} g(t) d t \\
& \leq \frac{1}{1-\tau_{*}} \int_{-\tau(0)}^{T-\tau(T)} \tilde{g}(t)|u(t)|_{\mathcal{H}}^{2} d t+\int_{0}^{T} g(t) d t \\
& \leq \frac{1}{1-\tau_{*}} \int_{-\tau(0)}^{T} \tilde{g}(t)|u(t)|_{\mathcal{H}}^{2} d t+\int_{0}^{T} g(t) d t,
\end{aligned}
$$

and therefore, taking into account that $\tilde{g} \in L^{p}(-\tau(0), T)$ for all $T>0$, we have that $\widetilde{F}(t, u(t-\tau(t)))$ belongs to $L^{2}(0, T ; \mathcal{H})$ for all $T>0$.

Therefore, from (17) we deduce that the derivative $u^{\prime}$ belongs to $L^{2}\left(0, T ; \mathcal{U}^{*}\right)$ for all $T>0$, and this fact and $u \in L^{2}(0, T ; \mathcal{U})$ for all $T>0$, imply that

$$
u \in C([0,+\infty) ; \mathcal{H}),
$$

and satisfies the energy equality

$$
\frac{d}{d t}|u(t)|_{\mathcal{H}}^{2}+2\langle\widetilde{A} u(t)+\widetilde{B}(u(t), u(t)), u(t)\rangle=2(\widetilde{F}(t, u(t-\tau(t))), u(t))_{\mathcal{H}},
$$

in the distributions sense on $(0,+\infty)$. 
Remark 5. If we define

$$
((u, v))_{\widetilde{A}}=\langle\widetilde{A} u, v\rangle, \quad \text { for all } u, v \in \mathcal{U},
$$

it is easy to see that $((\cdot, \cdot))_{\widetilde{A}}$ is a scalar product on $\mathcal{U}$ with associated norm equivalent to the norm $\|\cdot\|_{\mathcal{U}}$. From now on, without loss of generality, we suppose that

$$
((u, v))_{\mathcal{U}}=\langle\widetilde{A} u, v\rangle, \quad \text { for all } u, v \in \mathcal{U},
$$

and therefore,

$$
\lambda_{1}|u|_{\mathcal{H}}^{2} \leq\|u\|_{\mathcal{U}}^{2}, \quad \text { for all } u \in \mathcal{U}
$$

Remark 6. Recall that $D(\widetilde{A})$, the domain of $\widetilde{A}$, is the Hilbert space defined by

$$
D(\widetilde{A})=\{v \in \mathcal{U}: \widetilde{A} v \in \mathcal{H}\}
$$

with inner product

$$
(u, v)_{D(\widetilde{A})}:=(\widetilde{A} u, \widetilde{A} v) \quad u, v \in D(\widetilde{A}) .
$$

Theorem 7. Under the assumptions above, there exists a unique solution u to problem (17) and $u \in C([0,+\infty) ; \mathcal{H})$. Moreover, if $u^{0} \in \mathcal{U}$ then $u \in L^{2}(0, T ; D(\widetilde{A})) \cap$ $C([0, T] ; \mathcal{U})$ and $u^{\prime} \in L^{2}(0, T ; \mathcal{H})$ for all $T>0$.

Proof. We use the Galerkin method.

Step 1.- Construction of the approximating sequence. Consider the Hilbert

basis $\left\{v_{k}: k \geq 1\right\} \subset \mathcal{U}$ of $\mathcal{H}$ associated to the eigenvalues given by (15). For $m \geq 1$, let us denote $\mathcal{H}_{m}=\mathcal{U}_{m}$ the vector space spanned by $v_{1}, \ldots, v_{m}$. Let $u^{m}(t)=$ $\sum_{k=1}^{m} u_{m k}(t) v_{k}$, where $u_{m k}(t), k=1,2, \ldots, m$, are unknown functions, be the solution of the finite-dimensional problem

$$
\left\{\begin{array}{l}
\left(u^{m}(t), v_{k}\right)_{\mathcal{H}}+\int_{0}^{t}\left\langle\widetilde{A} u^{m}(s), v_{k}\right\rangle d s+\int_{0}^{t}\left\langle\widetilde{B}\left(u^{m}(s), u^{m}(s)\right), v_{k}\right\rangle d s \\
=\left(u^{0}, v_{k}\right)_{\mathcal{H}}+\int_{0}^{t}\left(\widetilde{F}\left(s, u^{m}(s-\tau(s))\right), v_{k}\right)_{\mathcal{H}} d s, t \geq 0,1 \leq k \leq m, \\
u^{m}(t)=\phi^{m}(t), \quad-h<t<0,
\end{array}\right.
$$

where $\phi^{m}(t)=P_{m} \phi(t)$, and $P_{m}$ is the orthogonal projection operator from $\mathcal{H}$ into $\mathcal{H}_{m}$.

From the assumptions on $\widetilde{A}, \widetilde{B}$ and $\widetilde{F}$, we know that there exists a unique local solution $u^{m}$ of (21) defined in $\left[0, t^{m}\right)$, with $0<t^{m} \leq+\infty$ (see for example [12]). That $t_{m}=+\infty$ will follow from the estimates below.

Step 2.-Estimates for the approximating sequence.

Let us fix $0<T<t_{m}$. Taking scalar product with $u^{m}(t)$ in (21), and using b1), c3), (19) and Young inequality, we deduce that

$$
\begin{aligned}
\frac{d}{d t}\left|u^{m}(t)\right|_{\mathcal{H}}^{2} & \leq-2|| u^{m}(t) \|_{\mathcal{U}}^{2}+\left|u^{m}(t)\right|_{\mathcal{H}}^{2}+\left|\widetilde{F}\left(t, u^{m}(t-\tau(t))\right)\right|_{\mathcal{H}}^{2} \\
& \leq-2|| u^{m}(t) \|_{\mathcal{U}}^{2}+\left|u^{m}(t)\right|_{\mathcal{H}}^{2}+g(t)\left|u^{m}(t-\tau(t))\right|_{\mathcal{H}}^{2}+g(t) .
\end{aligned}
$$


Integrating between 0 and $t \leq T$, and using the function $\tilde{g}$ defined in Remark 4 , we obtain

$$
\begin{aligned}
& \left|u^{m}(t)\right|_{\mathcal{H}}^{2}+2 \int_{0}^{t}\left\|u^{m}(s)\right\|_{\mathcal{U}}^{2} d s \\
& \leq\left|u^{0}\right|_{\mathcal{H}}^{2}+\int_{0}^{t}\left|u^{m}(s)\right|_{\mathcal{H}}^{2} d s+\frac{1}{1-\tau_{*}} \int_{-\tau(0)}^{t-\tau(t)} \tilde{g}(s)\left|u^{m}(s)\right|_{\mathcal{H}}^{2} d s+\int_{0}^{T} g(s) d s \\
& \leq K_{T}+\frac{1}{1-\tau_{*}} \int_{0}^{t}\left(\tilde{g}(s)+1-\tau_{*}\right)\left|u^{m}(s)\right|_{\mathcal{H}}^{2} d s,
\end{aligned}
$$

for all $t \in[0, T]$, where $K_{T}=\left|u^{0}\right|_{\mathcal{H}}^{2}+\frac{1}{1-\tau_{*}} \int_{-\tau(0)}^{0} \tilde{g}(s)|\phi(s)|_{\mathcal{H}}^{2} d s+\int_{0}^{T} g(s) d s$.

Thus, by Gronwall lemma,

$$
\left|u^{m}(t)\right|_{\mathcal{H}}^{2} \leq K_{T} \exp \left(\frac{1}{1-\tau_{*}} \int_{0}^{T}\left(\tilde{g}(s)+1-\tau_{*}\right) d s\right),
$$

for all $t \in[0, T]$.

From this inequality and (22) we deduce that $t_{m}=+\infty$ and

$$
\left\{u^{m}\right\} \text { is bounded in } L^{2}(0, T ; \mathcal{U}) \cap L^{\infty}(0, T ; \mathcal{H}) \text { for all } T>0 .
$$

Then, by a), b2) and Remark 4 we deduce that the sequences $\left\{\widetilde{A} u^{m}(t)\right\}$ and $\left\{\widetilde{B}\left(u^{m}(t), u^{m}(t)\right)\right\}$ are bounded in $L^{2}\left(0, T ; \mathcal{U}^{*}\right)$, and $\left\{\widetilde{F}\left(t, u^{m}(t-\tau(t))\right)\right\}$ is bounded in $L^{2}(0, T ; \mathcal{H})$, for all $T>0$.

Thus, taking into account that

$$
\frac{d}{d t} u^{m}(t)=P_{m}\left(-\widetilde{A} u^{m}(t)-\widetilde{B}\left(u^{m}(t), u^{m}(t)\right)+\widetilde{F}\left(t, u^{m}(t-\tau(t))\right)\right),
$$

we have that

$$
\left\{\frac{d}{d t} u^{m}\right\} \text { is bounded in } L^{2}\left(0, T ; \mathcal{U}^{*}\right) \text { for all } T>0 .
$$

\section{Step 3.- Taking limits in the finite-dimensional system.}

From the estimates above, and the compactness of the injection of $\mathcal{U}$ into $\mathcal{H}$, using in particular the compactness theorem 5.1 in chapter 1 of [14], we deduce that there exist a subsequence $\left\{u^{\mu}\right\} \subset\left\{u^{m}\right\}$ and a function $u \in L^{2 p^{\prime}}(-h, T ; \mathcal{H}) \cap L^{2}(0, T ; \mathcal{U}) \cap$ $L^{\infty}(0, T ; \mathcal{H})$, for all $T>0$, such that

$$
\begin{gathered}
u^{\mu} \rightarrow u \text { weak in } L^{2}(0, T ; \mathcal{U}) \cap L^{2}(-h, T ; \mathcal{H}), \\
u^{\mu} \stackrel{*}{*} u \text { weak } * \text { in } L^{\infty}(0, T ; \mathcal{H}), \\
u^{\mu} \rightarrow u \quad \text { in } L^{2}(0, T ; \mathcal{H}), \\
u^{\mu}(t) \rightarrow u(t) \quad \text { in } \mathcal{H} \text {, a.e. }-h<t<T,
\end{gathered}
$$

and

for all $T>0$.

$$
\frac{d}{d t} u^{\mu} \rightarrow \frac{d}{d t} u \text { weak in } L^{2}\left(0, T ; \mathcal{U}^{*}\right)
$$


Observe also that by (23) and the fact that $\left|\phi^{\mu}(t)\right|_{\mathcal{H}} \leq|\phi(t)|_{\mathcal{H}}$ a.e. $t \in(-h, 0)$, we have that there exists a function $f \in L^{2 p^{\prime}}(-h, T)$ for all $T>0$ such that

$$
\left|u^{\mu}(t)\right|_{\mathcal{H}} \leq f(t) \quad \text { a.e. }-h<t<+\infty .
$$

By c2), c3) (30) and (32), we can obtain by dominated convergence that

$$
\widetilde{F}\left(t, u^{\mu}(t-\tau(t))\right) \rightarrow \widetilde{F}(t, u(t-\tau(t))) \text { in } L^{2}(0, T ; \mathcal{H}),
$$

for all $T>0$.

On the other hand, evidently

$$
\widetilde{B}\left(u^{\mu}(t), u^{\mu}(t)\right)=\widetilde{B}\left(u^{\mu}(t)-u(t), u^{\mu}(t)\right)+\widetilde{B}\left(u(t), u^{\mu}(t)\right) .
$$

By b2), (29) and the fact that $\left\{u^{m}\right\}$ is bounded in $L^{2}(0, T ; \mathcal{U})$, we deduce that $\widetilde{B}\left(u^{\mu}(t)-u(t), u^{\mu}(t)\right) \rightarrow 0$ in $L^{1}\left(0, T ; \mathcal{U}^{*}\right)$, when $\mu \rightarrow+\infty$.

For the term $\widetilde{B}\left(u(t), u^{\mu}(t)\right)$, consider the mapping $\Psi: L^{2}(0, T ; \mathcal{U}) \rightarrow L^{2}\left(0, T ; \mathcal{U}^{*}\right)$ defined by

$$
\Psi(v)(t)=\widetilde{B}(u(t), v(t)) .
$$

From b2) and the fact that $u \in L^{\infty}(0, T ; \mathcal{H})$, it is clear that $\Psi$ is well defined, and is linear and continuous. Thus, as

$$
u^{\mu}(t) \rightarrow u(t) \text { weak in } L^{2}(0, T ; \mathcal{U}),
$$

we deduce that

$$
\widetilde{B}\left(u(t), u^{\mu}(t)\right) \rightarrow \widetilde{B}(u(t), u(t)) \text { weak in } L^{2}\left(0, T ; \mathcal{U}^{*}\right),
$$

for all $T>0$.

Consequently

$$
\widetilde{B}\left(u^{\mu}(t), u^{\mu}(t)\right) \rightarrow \widetilde{B}(u(t), u(t)) \text { weak in } L^{1}\left(0, T ; \mathcal{U}^{*}\right),
$$

for all $T>0$.

It is now standard to prove that $u$ is a solution of (17). The regularity of $u$ when $u^{0} \in \mathcal{U}$ can be proved also in a standard way by means of additional estimate on the sequence $\left\{u^{m}\right\}$ (see [15] for more details).

\section{Step 4.- Uniqueness.}

Let $u^{1}$ and $u^{2}$ be two solutions of (17) corresponding to the same initial data $u^{0}$ and $\phi$. Let us denote $\bar{u}=u^{1}-u^{2}$. Obviously,

$$
\bar{u}(t)=0 \text { in }(-h, 0) .
$$

On the other hand, by b1), one has

$$
\begin{aligned}
|\bar{u}(t)|_{\mathcal{H}}^{2} & +2 \int_{0}^{t}\langle\widetilde{A} \bar{u}(s), \bar{u}(s)\rangle d s+2 \int_{0}^{t}\left\langle\widetilde{B}\left(u^{1}(s), \bar{u}(s)\right), \bar{u}(s)\right\rangle d s \\
= & 2 \int_{0}^{t}\left(\widetilde{F}\left(s, u^{1}(s-\tau(s))\right)-\widetilde{F}\left(s, u^{2}(s-\tau(s))\right), \bar{u}(s)\right)_{\mathcal{H}} d s,
\end{aligned}
$$

for all $t \geq 0$. 
But, from b3) and Young's inequality,

$$
\begin{aligned}
& -2 \int_{0}^{t}\left\langle\widetilde{B}\left(u^{1}(s), \bar{u}(s)\right), \bar{u}(s)\right\rangle d s \\
& \leq 2 \int_{0}^{t}\|\bar{u}(s)\|_{\mathcal{U}}^{2} d s+\frac{c_{1}^{2}}{2} \int_{0}^{t}\left\|u^{1}(s)\right\|_{\mathcal{U}}^{2}|\bar{u}(s)|_{\mathcal{H}}^{2} d s .
\end{aligned}
$$

Let us fix $T>0$. We know that $u^{1}$ and $u^{2}$ belong to $C([0, T] ; \mathcal{H})$, thus there exists $R_{T}>0$ such that $\left|u^{1}(s)\right|_{\mathcal{H}} \leq R_{T}$ and $\left|u^{2}(s)\right|_{\mathcal{H}} \leq R_{T}$, for all $s \in[0, T]$. Consequently, by c2) and (35), we obtain

$$
\begin{aligned}
& 2 \int_{0}^{t}\left(\widetilde{F}\left(s, u^{1}(s-\tau(s))\right)-\widetilde{F}\left(s, u^{2}(s-\tau(s))\right), \bar{u}(s)\right)_{\mathcal{H}} d s \\
& \leq 2 L\left(R_{T}\right)\left(\int_{\{s \in(0, t): s-\tau(s)>0\}} g(s)\left|u^{1}(s-\tau(s))-u^{2}(s-\tau(s))\right|_{\mathcal{H}}^{2} d s\right)^{1 / 2} \times \\
& \times\left(\int_{0}^{t}|\bar{u}(s)|_{\mathcal{H}}^{2} d s\right)^{1 / 2} \\
& \leq \frac{2 L\left(R_{T}\right)}{\left(1-\tau_{*}\right)^{1 / 2}}\left(\int_{0}^{t} \tilde{g}(s)\left|u^{1}(s)-u^{2}(s)\right|_{\mathcal{H}}^{2} d s\right)^{1 / 2}\left(\int_{0}^{t}|\bar{u}(s)|_{\mathcal{H}}^{2} d s\right)^{1 / 2} \\
& \leq \frac{L\left(R_{T}\right)}{\left(1-\tau_{*}\right)^{1 / 2}} \int_{0}^{t}(\tilde{g}(s)+1)|\bar{u}(s)|_{\mathcal{H}}^{2} d s
\end{aligned}
$$

for all $t \in[0, T]$.

From (36), (37), (38) and (19), in particular we obtain

$$
|\bar{u}(t)|_{\mathcal{H}}^{2} \leq \int_{0}^{t}\left(\frac{L\left(R_{T}\right)}{\left(1-\tau_{*}\right)^{1 / 2}}(\tilde{g}(s)+1)+\frac{c_{1}^{2}}{2}\left\|u^{1}(s)\right\|_{\mathcal{U}}^{2}\right)|\bar{u}(s)|_{\mathcal{H}}^{2} d s,
$$

for all $t \in[0, T]$, and therefore, by the Gronwall lemma we deduce that $\bar{u}(t)=0$ for all $t \in[0, T]$.

\section{Asymptotic Behaviour of SOlutions}

In this section we obtain a result about the asymptotic behavior of the solutions of problem (17).

Theorem 8. Let us suppose that c2) holds with $g(t)=1$ for all $t \geq 0$, and there exist $a, b \geq 0$ such that

$$
|\widetilde{F}(t, u)|_{\mathcal{H}}^{2} \leq a|u|_{\mathcal{H}}^{2}+b \quad \forall u \in \mathcal{H}, \quad \forall t \geq 0 .
$$

Assume also that

$$
\lambda_{1}^{2}>\frac{a}{1-\tau_{*}}
$$

and let us denote $\varepsilon>0$ the unique solution of

$$
\lambda_{1}-\frac{a e^{\varepsilon h}}{\lambda_{1}\left(1-\tau_{*}\right)}-\varepsilon=0
$$

Then, for any $\left(u^{0}, \phi\right) \in \mathcal{H} \times L^{2}(-h, 0 ; \mathcal{H})$, the corresponding solution $u(t)$ of problem (17) satisfies

$$
|u(t)|_{\mathcal{H}}^{2} \leq\left\{\left|u^{0}\right|_{\mathcal{H}}^{2}+\frac{a e^{\varepsilon h}}{\lambda_{1}\left(1-\tau_{*}\right)} \int_{-h}^{0} e^{\varepsilon s}|\phi(s)|_{\mathcal{H}}^{2} d s\right\} e^{-\varepsilon t}+\frac{b}{\lambda_{1} \varepsilon}
$$


for all $t \geq 0$.

In particular, if $b=0$, then every solution $u(t)=u\left(t ; u^{0}, \phi\right)$ of (17) converges exponentially to 0 as $t \rightarrow+\infty$.

Proof. Let $u(t)$ the solution of problem (17) corresponding to the initial data $u^{0}, \phi$. We have

$$
\begin{aligned}
\frac{d}{d t}\left(e^{\varepsilon t}|u(t)|_{\mathcal{H}}^{2}\right) & =\varepsilon e^{\varepsilon t}|u(t)|_{\mathcal{H}}^{2}+e^{\varepsilon t} \frac{d}{d t}|u(t)|_{\mathcal{H}}^{2} \\
& =\varepsilon e^{\varepsilon t}|u(t)|_{\mathcal{H}}^{2}-2 e^{\varepsilon t}\|u(t)\|_{\mathcal{U}}^{2}+2 e^{\varepsilon t}(\widetilde{F}(t, u(t-\tau(t))), u(t))_{\mathcal{H}} \\
& \leq \varepsilon e^{\varepsilon t}|u(t)|_{\mathcal{H}}^{2}-2 e^{\varepsilon t}\|u(t)\|_{\mathcal{U}}^{2}+\frac{e^{\varepsilon t}}{\lambda_{1}}|\widetilde{F}(t, u(t-\tau(t)))|_{\mathcal{H}}^{2}+e^{\varepsilon t} \lambda_{1}|u(t)|_{\mathcal{H}}^{2}
\end{aligned}
$$

Integrating, we obtain,

$$
\begin{aligned}
e^{\varepsilon t}|u(t)|_{\mathcal{H}}^{2} & \leq\left|u^{0}\right|_{\mathcal{H}}^{2}+\left(\varepsilon \lambda_{1}^{-1}-1\right) \int_{0}^{t} e^{\varepsilon s}\|u(s)\|_{\mathcal{U}}^{2} d s \\
& +\lambda_{1}^{-1} \int_{0}^{t} e^{\varepsilon s}|\widetilde{F}(s, u(s-\tau(s)))|_{\mathcal{H}}^{2} d s .
\end{aligned}
$$

By (39),

$$
\begin{aligned}
\int_{0}^{t} e^{\varepsilon s}|\widetilde{F}(s, u(s-\tau(s)))|_{\mathcal{H}}^{2} d s & \leq \int_{0}^{t} e^{\varepsilon s}\left(a|u(s-\tau(s))|_{\mathcal{H}}^{2}+b\right) d s \\
& \leq \frac{a e^{\varepsilon h}}{1-\tau_{*}} \int_{-\tau(0)}^{t} e^{\varepsilon s}|u(s)|_{\mathcal{H}}^{2} d s+\frac{b e^{\varepsilon t}}{\varepsilon} \\
& \leq \frac{a e^{\varepsilon h}}{1-\tau_{*}} \int_{-h}^{0} e^{\varepsilon s}|\phi(s)|_{\mathcal{H}}^{2} d s+\frac{a e^{\varepsilon h}}{1-\tau_{*}} \int_{0}^{t} e^{\varepsilon s}|u(s)|_{\mathcal{H}}^{2} d s \\
& +\frac{b e^{\varepsilon t}}{\varepsilon} .
\end{aligned}
$$

Thus,

$$
\begin{aligned}
e^{\varepsilon t}|u(t)|_{\mathcal{H}}^{2} & \leq\left|u^{0}\right|_{\mathcal{H}}^{2}+\lambda_{1}^{-1}\left(\varepsilon-\lambda_{1}+\frac{a e^{\varepsilon h}}{\lambda_{1}\left(1-\tau_{*}\right)}\right) \int_{0}^{t} e^{\varepsilon s}\|u(s)\|_{\mathcal{U}}^{2} d s \\
& +\frac{a e^{\varepsilon h}}{\lambda_{1}\left(1-\tau_{*}\right)} \int_{-h}^{0} e^{\varepsilon s}|\phi(s)|_{\mathcal{H}}^{2} d s+\frac{b e^{\varepsilon t}}{\lambda_{1} \varepsilon} .
\end{aligned}
$$

By the definition of $\varepsilon$, we obtain (41).

\section{Convergence to the stationary solutions of the ABStract Problem}

We first obtain existence, and eventual uniqueness, of stationary solutions of the abstract problem. Then we prove a result about exponential convergence of the solutions of (17) to a stationary solution.

From now on we assume that $\widetilde{F}: \mathcal{H} \longrightarrow \mathcal{H}$ is independent of $t$, and satisfies

$$
|\widetilde{F}(u)|_{\mathcal{H}}^{2} \leq a|u|_{\mathcal{H}}^{2}+b \quad \forall u \in \mathcal{H},
$$

with $a, b \geq 0$. 
4.1. Existence of stationary solutions. Consider the equation

$$
\frac{d u}{d t}+\widetilde{A} u+\widetilde{B}(u, u)=\widetilde{F}(u(t-\tau(t))) .
$$

Definition 9. It is said that $u_{\infty} \in \mathcal{U}$ is a stationary solution of (43) if it satisfies

$$
\widetilde{A} u_{\infty}+\widetilde{B}\left(u_{\infty}, u_{\infty}\right)=\widetilde{F}\left(u_{\infty}\right) .
$$

Remark 10. Observe that by b3), if $u_{\infty} \in \mathcal{U}$ is a stationary solution of (43) then in fact $u_{\infty} \in D(\widetilde{A})$.

Observe also that

$$
\begin{aligned}
& \left\|u_{\infty}\right\|_{\mathcal{U}}^{2}=\left(\widetilde{F}\left(u_{\infty}\right), u_{\infty}\right)_{\mathcal{H}} \leq\left|\widetilde{F}\left(u_{\infty}\right)\right|_{\mathcal{H}}\left|u_{\infty}\right|_{\mathcal{H}} \\
& \leq a^{1 / 2}\left|u_{\infty}\right|_{\mathcal{H}}^{2}+b^{1 / 2}\left|u_{\infty}\right|_{\mathcal{H}} \\
& \leq a^{1 / 2} \lambda_{1}^{-1}\left\|u_{\infty}\right\|_{\mathcal{U}}^{2}+b^{1 / 2} \lambda_{1}^{-1 / 2}\left\|u_{\infty}\right\|_{\mathcal{U}}
\end{aligned}
$$

Thus, if $\lambda_{1}>a^{1 / 2}$, then every stationary solution $u_{\infty} \in \mathcal{U}$ of (43) satisfies

$$
\left\|u_{\infty}\right\|_{\mathcal{U}} \leq \frac{b^{1 / 2} \lambda_{1}^{1 / 2}}{\lambda_{1}-a^{1 / 2}}=\widetilde{C} .
$$

Theorem 11. Suppose that $\widetilde{F}: \mathcal{H} \longrightarrow \mathcal{H}$ is continuous and satisfies (42) with $\lambda_{1}>a^{1 / 2}$. Then, there exists a stationary solution of (43).

If, in addition, $\widetilde{F}$ is locally Lipschitz and

$$
\lambda_{1}-c_{1} \widetilde{C} \lambda_{1}^{1 / 2}>L(\widetilde{C}),
$$

where $c_{1}$ is the constant appearing in b2), $\widetilde{C}$ is given by (46), and $L(\widetilde{C})$ is the Lipschitz constant for $\widetilde{F}$ associated to $\widetilde{C}$, then the stationary solution of (43) is unique.

Proof. The proof of the existence follows the same lines as those used in [4] (see also [15]).

As for the uniqueness when $\widetilde{F}$ is locally Lipschitz and satisfies (47), assume that $u_{\infty}$ and $\widetilde{u}_{\infty}$ are two stationary solutions of (43). Then,

$$
\begin{aligned}
& \left\langle\widetilde{A} u_{\infty}-\widetilde{A} \widetilde{u}_{\infty}, v\right\rangle+\left\langle\widetilde{B}\left(u_{\infty}, u_{\infty}\right)-\widetilde{B}\left(\widetilde{u}_{\infty}, \widetilde{u}_{\infty}\right), v\right\rangle \\
& =\left(\widetilde{F}\left(u_{\infty}\right)-\widetilde{F}\left(\widetilde{u}_{\infty}\right), v\right)_{\mathcal{H}}, \forall v \in \mathcal{U} .
\end{aligned}
$$

Taking $v=u_{\infty}-\widetilde{u}_{\infty}$ in (48), from (19), b1) and b3), we obtain

$$
\begin{aligned}
& \left\|u_{\infty}-\widetilde{u}_{\infty}\right\|_{\mathcal{U}}^{2} \\
& =-\left\langle\widetilde{B}\left(u_{\infty}, u_{\infty}\right)-\widetilde{B}\left(\widetilde{u}_{\infty}, \widetilde{u}_{\infty}\right), u_{\infty}-\widetilde{u}_{\infty}\right\rangle+\left(\widetilde{F}\left(u_{\infty}\right)-\widetilde{F}\left(\widetilde{u}_{\infty}\right), u_{\infty}-\widetilde{u}_{\infty}\right)_{\mathcal{H}} \\
& =-\left\langle\widetilde{B}\left(\widetilde{u}_{\infty}, u_{\infty}-\widetilde{u}_{\infty}\right), u_{\infty}-\widetilde{u}_{\infty}\right\rangle+\left(\widetilde{F}\left(u_{\infty}\right)-\widetilde{F}\left(\widetilde{u}_{\infty}\right), u_{\infty}-\widetilde{u}_{\infty}\right)_{\mathcal{H}} \\
(49) \quad & \leq c_{1}\left\|\widetilde{u}_{\infty}\right\|_{\mathcal{U}}\left\|u_{\infty}-\widetilde{u}_{\infty}\right\|_{\mathcal{U}}\left|u_{\infty}-\widetilde{u}_{\infty}\right|_{\mathcal{H}}+\left(\widetilde{F}\left(u_{\infty}\right)-\widetilde{F}\left(\widetilde{u}_{\infty}\right), u_{\infty}-\widetilde{u}_{\infty}\right)_{\mathcal{H}} .
\end{aligned}
$$

Thus, taking into account (46), we deduce from (49) that

$$
\left\|u_{\infty}-\widetilde{u}_{\infty}\right\|_{\mathcal{U}}^{2} \leq\left(c_{1} \widetilde{C} \lambda_{1}^{-1 / 2}+L(\widetilde{C}) \lambda_{1}^{-1}\right)\left\|u_{\infty}-\widetilde{u}_{\infty}\right\|_{\mathcal{U}}^{2} .
$$

But the assumption (47) is equivalent to

$$
c_{1} \widetilde{C} \lambda_{1}^{-1 / 2}+L(\widetilde{C}) \lambda_{1}^{-1}<1,
$$

and therefore the uniqueness of stationary solution follows from (50). 


\subsection{Exponential convergence to a stationary solution.}

Theorem 12. Suppose that $\widetilde{F}: \mathcal{H} \rightarrow \mathcal{H}$ satisfies (42) and is locally Lipschitz continuous, i.e., satisfies c2) with $g(t) \equiv 1$. Suppose also that

$$
\lambda_{1}>a^{1 / 2} \quad \text { and } \quad \lambda_{1}^{2}>\frac{a}{1-\tau_{*}},
$$

and, for each $M>0$, let us define

$$
\rho(M)=M\left(1+\frac{a e^{\varepsilon h}}{\lambda_{1}\left(1-\tau_{*}\right)}\right)+\frac{b}{\lambda_{1} \varepsilon},
$$

where $\varepsilon>0$ is defined by (40).

Let $u_{\infty} \in \mathcal{U}$ be a stationary solution of (43), and suppose that there exists $M>0$ such that

$$
\left|u_{\infty}\right|_{\mathcal{H}} \leq M \quad \text { and } \quad \lambda_{1}>\frac{2 \lambda_{1} c_{1} b^{1 / 2}}{\lambda_{1}-a^{1 / 2}}+\frac{L(\rho(M))}{\lambda_{1}\left(1-\tau_{*}\right)} .
$$

Then, there exists $\lambda=\lambda(M)>0$ such that for any $u^{0}, \phi$, satisfying $\left|u^{0}\right|_{\mathcal{H}}^{2} \leq$ $M,\|\phi\|_{L^{2}(-h, 0 ; \mathcal{H})}^{2} \leq M$, the corresponding solution $u(t)=u\left(t ; u^{0}, \phi\right)$ of $(17)$ satisfies

$$
\begin{aligned}
& \left|u(t)-u_{\infty}\right|_{\mathcal{H}}^{2} \\
& \leq\left\{\left|u^{0}-u_{\infty}\right|_{\mathcal{H}}^{2}+\frac{e^{\lambda h}}{\lambda_{1}\left(1-\tau_{*}\right)} \int_{-h}^{0} e^{\lambda s}\left|\widetilde{F}(\phi(s))-\widetilde{F}\left(u_{\infty}\right)\right|_{\mathcal{H}}^{2} d s\right\} e^{-\lambda t \quad \text { for all } t \geq 0 .}
\end{aligned}
$$

Proof. Let $M>0$ satisfy (53), and consider $u^{0}, \phi$, satisfying $\left|u^{0}\right|_{\mathcal{H}}^{2} \leq M$, and $\|\phi\|_{L^{2}(-h, 0 ; \mathcal{H})}^{2} \leq M$. Let $u(t)=u\left(t ; u^{0}, \phi\right)$ be the corresponding solution of (17), and denote $w(t)=u(t)-u_{\infty}$. We have

$$
\frac{d}{d t} w(t)+\widetilde{A} w(t)+\widetilde{B}(u(t), u(t))-\widetilde{B}\left(u_{\infty}, u_{\infty}\right)=\widetilde{F}(u(t-\tau(t)))-\widetilde{F}\left(u_{\infty}\right) .
$$

Then, for any $\lambda>0$ one has

$$
\begin{aligned}
\frac{d}{d t}\left(e^{\lambda t}|w(t)|_{\mathcal{H}}^{2}\right) & =\lambda e^{\lambda t}|w(t)|_{\mathcal{H}}^{2}+e^{\lambda t} \frac{d}{d t}|w(t)|_{\mathcal{H}}^{2} \\
& \leq \lambda e^{\lambda t}|w(t)|_{\mathcal{H}}^{2}-2 e^{\lambda t}|| w(t) \|_{\mathcal{U}}^{2}+2 e^{\lambda t}\left\langle\widetilde{B}\left(u_{\infty}, w(t)\right), w(t)\right\rangle \\
& +2 e^{\lambda t}\left|\widetilde{F}(u(t-\tau(t)))-\widetilde{F}\left(u_{\infty}\right)\right|_{\mathcal{H}}|w(t)|_{\mathcal{H}} \\
& \leq \lambda e^{\lambda t}|w(t)|_{\mathcal{H}}^{2}-2 e^{\lambda t}|| w(t) \|_{\mathcal{U}}^{2}+2 e^{\lambda t}\left|\left\langle\widetilde{B}\left(u_{\infty}, w(t)\right), w(t)\right\rangle\right| \\
& +\frac{e^{\lambda t}}{\lambda_{1}}\left|\widetilde{F}(u(t-\tau(t)))-\widetilde{F}\left(u_{\infty}\right)\right|_{\mathcal{H}}^{2}+e^{\lambda t} \lambda_{1}|w(t)|_{\mathcal{H}}^{2}
\end{aligned}
$$

By b3),

$$
\begin{aligned}
\left|\left\langle\widetilde{B}\left(u_{\infty}, w(t)\right), w(t)\right\rangle\right| & \leq c_{1}\left\|u_{\infty}\right\| \mathcal{U}\|w(t)\|_{\mathcal{U}}|w(t)|_{\mathcal{H}} \\
& \leq c_{1} \lambda_{1}^{-1 / 2}\|w(t)\|_{\mathcal{U}}^{2}\left\|u_{\infty}\right\|_{\mathcal{U}}
\end{aligned}
$$


and therefore, from (46) and (20) we obtain

$$
\begin{aligned}
\frac{d}{d t}\left(e^{\lambda t}|w(t)|_{\mathcal{H}}^{2}\right) & \leq \frac{e^{\lambda t}}{\lambda_{1}}\left(\lambda-\lambda_{1}+\frac{2 c_{1} \lambda_{1} b^{1 / 2}}{\lambda_{1}-a^{1 / 2}}\right)\|w(t)\|_{\mathcal{U}}^{2} \\
& +\frac{e^{\lambda t}}{\lambda_{1}}\left|\widetilde{F}(u(t-\tau(t)))-\widetilde{F}\left(u_{\infty}\right)\right|_{\mathcal{H}}^{2} .
\end{aligned}
$$

Integrating this inequality, we obtain

$$
\begin{aligned}
e^{\lambda t}|w(t)|_{\mathcal{H}}^{2} & \leq|w(0)|_{\mathcal{H}}^{2}+\lambda_{1}^{-1}\left(\lambda-\lambda_{1}+\frac{2 c_{1} \lambda_{1} b^{1 / 2}}{\lambda_{1}-a^{1 / 2}}\right) \int_{0}^{t} e^{\lambda s}\|w(s)\|_{\mathcal{U}}^{2} d s \\
& +\lambda_{1}^{-1} \int_{0}^{t} e^{\lambda s}\left|\widetilde{F}(u(s-\tau(s)))-\widetilde{F}\left(u_{\infty}\right)\right|_{\mathcal{H}}^{2} d s \\
& \leq|w(0)|_{\mathcal{H}}^{2}+\lambda_{1}^{-1}\left(\lambda-\lambda_{1}+\frac{2 c_{1} \lambda_{1} b^{1 / 2}}{\lambda_{1}-a^{1 / 2}}\right) \int_{0}^{t} e^{\lambda s}\|w(s)\|_{\mathcal{U}}^{2} d s \\
& +\frac{\lambda_{1}^{-1} e^{\lambda h}}{1-\tau *} \int_{-h}^{0} e^{\lambda s}\left|\widetilde{F}(\phi(s))-\widetilde{F}\left(u_{\infty}\right)\right|_{\mathcal{H}}^{2} d s \\
& +\frac{\lambda_{1}^{-1} e^{\lambda h}}{1-\tau *} \int_{0}^{t} e^{\lambda s}\left|\widetilde{F}(u(s))-\widetilde{F}\left(u_{\infty}\right)\right|_{\mathcal{H}}^{2} d s .
\end{aligned}
$$

On the other hand, by (41), we have that in particular

$$
|u(t)|_{\mathcal{H}}^{2} \leq\left|u^{0}\right|_{\mathcal{H}}^{2}+\frac{a e^{\varepsilon h}}{\lambda_{1}\left(1-\tau_{*}\right)} \int_{-h}^{0} e^{\varepsilon s}|\phi(s)|_{\mathcal{H}}^{2} d s+\frac{b}{\lambda_{1} \varepsilon},
$$

for all $t \geq 0$, and consequently, as $\left|u^{0}\right|_{\mathcal{H}}^{2} \leq M,\|\phi\|_{L^{2}(-h, 0 ; \mathcal{H})}^{2} \leq M$, by (52) we have

$$
|u(t)|_{\mathcal{H}} \leq \rho(M), \quad \forall t \geq 0 .
$$

From (56) and the local Lipschitz continuity of $\widetilde{F}$, we obtain

$$
\begin{aligned}
e^{\lambda t}|w(t)|_{\mathcal{H}}^{2} & \leq|w(0)|_{\mathcal{H}}^{2}+\lambda_{1}^{-1}\left(\lambda-\lambda_{1}+\frac{2 c_{1} \lambda_{1} b^{1 / 2}}{\lambda_{1}-a^{1 / 2}}+\frac{e^{\lambda h} L(\rho(M))}{\lambda_{1}(1-\tau *)}\right) \int_{0}^{t} e^{\lambda s}\|w(s)\|_{\mathcal{U}}^{2} d s \\
& +\frac{\lambda_{1}^{-1} e^{\lambda h}}{1-\tau *} \int_{-h}^{0} e^{\lambda s}\left|\widetilde{F}(\phi(s))-\widetilde{F}\left(u_{\infty}\right)\right|_{\mathcal{H}}^{2} d s .
\end{aligned}
$$

By (53), there exists $\lambda>0$ such that

$$
\lambda-\lambda_{1}+\frac{2 c_{1} \lambda_{1} b^{1 / 2}}{\lambda_{1}-a^{1 / 2}}+\frac{e^{\lambda h} L(\rho(M))}{\lambda_{1}(1-\tau *)} \leq 0,
$$

and for this $\lambda$ we thus obtain (54).

Remark 13. Suppose that the Lipschitz constant L appearing in c2) is such that $L(R) \rightarrow 0$ as $R \rightarrow 0$. We know that $\left|u_{\infty}\right| \leq \frac{b^{1 / 2}}{\lambda_{1}-a^{1 / 2}}$. If we take $M=\frac{b^{1 / 2}}{\lambda_{1}-a^{1 / 2}}$, then $M \rightarrow 0$ provided that $b \rightarrow 0$ and, therefore, $\rho(M) \rightarrow 0$, and $L(\rho(M)) \rightarrow 0$. Consequently, for this choice of $M$ the assumption (53) holds if $b>0$ is small enough.

Remark 14. If (53) is satisfied for the choice of $M=\frac{b^{1 / 2}}{\lambda_{1}-a^{1 / 2}}$, then the stationary solution is unique. 


\section{Applichtion to the $\alpha$-Navier-Stokes model (1)}

Let us now establish some results concerning our model (1). As can be easily checked, the abstract theory developed in the previous sections can be applied to our model (1), by simply taking into account the relationships existing between the constants appearing in the abstract form (17), and in our motivating model (1). We will only state some of the results concerning the existence and asymptotic behaviour of the solutions to (1).

Theorem 15. Suppose that d1)-d4) are fulfilled, and that $\tau \in C^{1}([0,+\infty)$, with $0 \leq \tau(t) \leq h$ for all $t \geq 0$, and that (10) holds. Then, there exists a unique variational solution of (1). Moreover, $u \in C([0,+\infty) ; V)$, and if $u^{0} \in D(A)$, then $u \in L^{2}(0, T ; D(A)) \cap C([0, T] ; D(A))$ and $u^{\prime} \in L^{2}(0, T ; V)$ for all $T>0$.

Proof. Observe that, under the assumptions in our theorem, the hypotheses in Theorem 7 are fulfilled.

Theorem 16. Let us suppose that d2) holds with $g(t)=1$ for all $t \geq 0$, and there exist $a, b \geq 0$ such that

$$
\|F(t, u)\|^{2} \leq a\|u\|^{2}+b \quad \forall u \in V, \quad \forall t \geq 0 .
$$

Assume also that

$$
\nu^{2} \mu_{1}^{2}>\frac{a}{1-\tau_{*}},
$$

and let us denote $\varepsilon>0$ the unique solution of

$$
\nu \mu_{1}-\frac{a e^{\varepsilon h}}{\nu \mu_{1}\left(1-\tau_{*}\right)}-\varepsilon=0
$$

Then, for any $\left(u^{0}, \phi\right) \in V \times L^{2}(-h, 0 ; V)$, the corresponding solution $u(t)$ of problem (1) satisfies

$$
\|u(t)\|^{2} \leq\left\{\left\|u^{0}\right\|^{2}+\frac{a e^{\varepsilon h}}{\nu \mu_{1}\left(1-\tau_{*}\right)} \int_{-h}^{0} e^{\varepsilon s}\|\phi(s)\|^{2} d s\right\} e^{-\varepsilon t}+\frac{b}{\nu \mu_{1} \varepsilon},
$$

for all $t \geq 0$.

In particular, if $b=0$, then every solution $u(t)=u\left(t ; u^{0}, \phi\right)$ of (1) converges exponentially in $V$ to 0 as $t \rightarrow+\infty$.

Proof. Observe that d2) with $g(t)=1$ implies c2) with $g(t)=1$. On the other hand, (58) implies (39). Then, Theorem 8 ensures the assertions in our theorem.

Remark 17. In a similar way, one can obtain analogous results to Theorem 11 and Theorem 12, but for our primitive model (1). We leave the details to the reader.

\section{FinAl COMMENTS, CONCLUSIONS, AND REMARKS}

We have first developed an abstract theory for an evolution equation with variable delay in the forcing term. Next, we have applied the abstract theory to the particular example of the three-dimensional $\alpha$-Navier-Stokes model with locally Lipschitz forcing term with some kind of memory (namely, with variable delay). We point out that our abstract formulation includes other important types of partial differential equations (for instance, if $\widetilde{B}=0$, our abstract model becomes a reaction-diffusion equation with delays). As we mentioned in the Introduction, although it is possible to develop a theory for a more general delay term (written, for instance, in a 
functional form), we have chosen this particular type of delay motivated by several reasons. On the one hand, the set of assumptions for the analysis is more clearly understood in this case (for the functional setting, we need additional assumptions, in integral form, similar to the ones in [6] and [5]). On the other, this kind of delay was considered by Taniguchi [20] for the two-dimensional Navier-Stokes equations. To be more precise, the delay term $f(t-\tau(t), u(t-\tau(t)))$ in [20] is a particular case of our term $f(t, u(t-\tau(t)))$ in this paper. Moreover, the technique used in [20] is based in considering a Lyapunov functional $W\left(t, u_{t}\right)$, while in our case it is not necessary to construct such a functional, but only proceed in a more direct way.

Acknowledgements. We would like to thank the referees for their interesting and helpful suggestions and comments.

\section{REFERENCES}

[1] T. Caraballo, I.D. Chueshov, P. Marín-Rubio, J. Real, Existence and asymptotic behaviour for stochastic heat equations with multiplicative noise in materials with memory, Discrete and Continuous Dynamical Systems, Series A, 18 (2007), 253-270

[2] T. Caraballo, P.E. Kloeden, J. Real, Unique strong solutions and V-attractors fo a three dimensional system of globally modified Navier-Stokes equations, Advanced Nonlinear Studies 6 (2006), 411-436.

[3] T. Caraballo, P.E. Kloeden, J. Real, Invariant measures and statistical solutions of the globally modified Navier-Stokes equations, Discrete Contin. Dyn. Syst. B 10 (2008), 761-781.

[4] T. Caraballo, A.M. Márquez-Durán, J. Real, On the asymptotic behaviour of a stochastic 3D LANS- $\alpha$ model, Appl. Math. Optim. 53 (2006), 141-161.

[5] T. Caraballo, A.M. Márquez-Durán and J. Real, Asymptotic behaviour of the threedimensional $\alpha$-Navier-Stokes model with delays, J. Math. Anal. Appl. 340 (2008), 410-423.

[6] T. Caraballo, J. Real, Navier-Stokes equations with delays, R. Soc. Lond. Proc. Ser. A Math. Phys. Eng. Sci. 457 (2001), 2441-2453.

[7] T. Caraballo, J. Real, Attractors for 2D-Navier-Stokes models with delays, J. Differential Equations 205 (2004), 270-296.

[8] T. Caraballo, J. Real, T. Taniguchi, The existence and uniqueness of solutions to stochastic 3dimensional lagrangian averaged Navier-Stokes equations, Proc. R. Soc. Lond. Ser. A Math. Phys. Eng. Sci. 462 (2006), 459-479.

[9] P. Constantin, Near identity transformations for the Navier-Stokes equations, in Handbook of Mathematical Fluid Dynamics, Vol. II, 117-141, North-Holland, Amsterdam, 2003.

[10] D. Coutand, J. Peirce, S. Shkoller, Global well-posedness of weak solutions for the Lagrangian averaged Navier-Stokes equations on bounded domains, Comm. on Pure and Appl. Anal. 1 (2002), 35-50.

[11] C. Foias, D.D. Holm, E.S. Titi, The three dimensional viscous Camassa-Holm equations and their relation to the Navier-Stokes equations and turbulence theory, J. Dyn. Diff. Eq. 14 (2002), 1-35.

[12] J.K. Hale, S.M. Verduyn Lunel, Introduction to Functional Differential Equations, Springer Verlag, New York, 1993.

[13] A.A. Ilyin, E.S. Titi, Attractors for the two-dimensional Navier-Stokes- $\alpha$ model: an $\alpha$ dependence study, J. Dynamic Differential Equations 15 (2003), 751-778.

[14] J.L. Lions, Quelques méthodes de résolutions des problèmes aux limites non linéaires, Dunod Gauthier-Villars, (1969).

[15] A.M. Márquez Durán, PhD Thesis, Universidad de Sevilla, July 2005.

[16] J.E. Marsden, S. Shkoller, Global well-posedness for the LANS- $\alpha$ equations on bounded domains, R. Soc. Lond. Philos. Trans. Ser. A Math. Phys. Eng. Sci. 359 (2001), 1449-1468.

[17] J. D. Murray, Mathematical Biology, Berlin: Springer-Verlag, 1993.

[18] W. Ruess, Existence of solutions to partial functional differential equations with delay, in Theory and Applications of Nonlinear Operators of Accretive and Monotone Type, (ed. A.G. Kartsatos), Marcel Dekker, New York (1996), 259-288. 
[19] W. Ruess, Existence and stability of solutions to partial functional differential equations with delay, Adv. Diff. Eqns. 4 (6) (1999), 843-876.

[20] T. Taniguchi, The exponential behaviour of Navier-Stokes equations with time delay external force, Discrete Contin. Dyn. Syst. 12 (2005), no. 5, 997-1018

[21] J. Wu, Theory and applications of partial functional differential equations, Springer-Verlag, New York (1996).

(T. Caraballo) Dpto. Ecuaciones Diferenciales y Análisis Numérico, Universidad de Sevilla, Apdo. de Correos 1160, 41080-Sevilla (Spain),

E-mail address, T. Caraballo: caraball@us.es

(A.M. Márquez-Durán) Dpto. Economía, MÉtodos Cuantitativos e Historia Económica, Universidad Pablo de Olavide, Edificio No 3, José Moñino, Ctra. de Utrera, Km. 141013 Sevilla, (Spain),

E-mail address, A.M. Márquez-Durán: ammardur@upo.es

(J. Real) Dpto. Ecuaciones Diferenciales y AnÁlisis Numérico, Universidad de Sevilla, Apdo. de Correos 1160, 41080-Sevilla (Spain),

E-mail address, J. Real: jreal@us.es 